\title{
Clinical Results of Surgically Treated Medial Humeral Epicondylar Apophyseal Avulsion Injury in Children and Adolescent
}

\author{
Ruban Raj Joshi, ${ }^{\mathrm{acc}}$ Gabriel David Sundararaj ${ }^{\mathrm{b}, \mathrm{c}}$
}

\begin{abstract}
:
Introduction: Fractures of the medial humeral epicondyle represent approximately $10 \%$ of all paediatric elbow fractures. Objective of our study was to assess treatment outcomes of children and adolescent with medial epicondylar fracture of the elbow using standard operative protocols. Methods: Twenty surgically treated fractures of the medial humeral epicondyle were analysed \& reviewed for their epidemiological, clinical and surgical parameters. A valgus stress test was performed under general anesthesia or sedation. All patients underwent open reduction internal fixation using a similar technique. The medial epicondylar fragment was anatomically reduced and fixed in all cases with screws, Kirshner wires or tension band wiring. At final evaluation, union (radiologically) and elbow function [MAYO elbow performance score (MEPS)] was assessed. Results: An evaluation of all of our patients after a mean follow-up of 8.75 months $(S D=4.76)$ after initial surgery was possible. The mean age of patients at the time of injury was 10.8 years $(S D=2.3)$. Fifteen $(75 \%)$ dominant elbows were injured in our study and $12(60 \%)$ elbows had an associated elbow dislocation. On examination in operating room post anaesthesia, all of the elbow injuries revealed some degree of valgus instability. All of our patients( $\mathrm{n}=20)$ showed good to excellent results in the MAYO elbow performance score (MEPS). Radiographically, union was achieved in all cases. Three patients developed postoperative ulnar nerve neuropraxia, all recovered at time of final follow up. One patient developed mild lateral heterotrophic ossification but did not require any additional surgical intervention. Conclusion: Our results suggest that open reduction internal fixation of displaced medial epicondyle fractures leads to satisfactory motion and function. A valgus stress test in operating room can reveal the true nature of joint instability that can warrant operative stabilization of medial epicondylar injuries.
\end{abstract}

Keywords: children • dislocation • humerus $\bullet$ internal fixation • open reduction

\section{INTRODUCTION:}

Medial epicondylar avulsion fractures account for $11-20 \%$ of pediatric elbow injuries and $1.3 \%$ of all fractures in children. ${ }^{1}$ This type of fractures occurs typically between ages of 9 and

\footnotetext{
a - Lecturer

b - Professor and Head

c- Department of Orthopaedic Surgery \& Traumatology,

Lumbini Medical College Teaching Hospital, Palpa, Nepal
}

Corresponding Author:

Dr. Ruban Raj Joshi

e-mail: rubanjoshi@hotmail.com

How to cite this article:

Joshi RR, Sundararaj GD. Clinical results of surgically treated medial humeral epicondylar apophyseal avulsion injury in children and adolescent. Journal of Lumbini Medical College. 2014;2(2):31-6. doi: 10.22502/jlmc.v2i2.54.
14 and boys are four times more affected. ${ }^{2}$ Half of the cases are associated with an acute traumatic elbow dislocation and intra articular incarceration of medial epicondylar fragment within the elbow occurs in $15-18 \%$ of children. ${ }^{2} 10-16 \%$ of children with these injuries are associated with ulnar nerve dysfunction. ${ }^{2,3}$

There is a wide agreement on conservative treatment for undisplaced or minimally displaced medial epicondyle fractures. ${ }^{4}$ Such injuries are simply treated with immobilization and early motion to obviate stiffness. ${ }^{5}$ However opinions differ regarding management of displaced medial epicondyle fractures. Moreover, the definition of displacement varies between studies; some denominate the displaced fractures if $>2 \mathrm{~mm}$, but others, rely on $>5 \mathrm{~mm}^{1}{ }^{1}$ Some literatures testify excellent to good results with conservative 
management, but above $60 \%$ of patients demonstrate radiographic nonunion that may lead to valgus instability. ${ }^{4}$ Here, we present 20 cases of surgically managed elbow medial epicondyle avulsion injuries in children and adolescents. We aimed to characterize functional outcome, range of motion, ulnar nerve dysfunction, heterotopic ossification, and any other complications.

\section{METHODS:}

In this retrospective study between October 2011 and October 2014 from Lumbini Medical College Teaching Hospital, 28 children (18 boys and 10 girls) with medial epicondyle fractures were reviewed. We excluded eight children with type-I fractures and selected 20 displaced medial epicondyle fractures that were surgically treated. Clinical signs of anteromedial ecchymosis were recorded and documented. Under general anesthesia, a valgus stress test was performed which was considered positive when there was medial laxity and instability based on further displacement of the fracture. Radiographic analysis was done using plain elbow posteroanterior, lateral and oblique views which provided information regarding fracture anatomy, its displacement, whether incarcerated in the joint and whether associated with elbow dislocation.

On the basis of extent of displacement and articular instability, four types of medial epicondyle fractures are distinguished.

1. Type-I: with no or minimal displacement (excluded of this study).

2. Type-II: moderate displacement, of more than half of the metaphyseal fracture area and a positive valgus stress test.

3. Type-III: major displacement associated with obvious dislocation of the elbow or dislocation during the valgus stress test; the medial epicondyle remains at the level of the articular line.

4. Type-IV: posterior displacement of the epicondyle with intra-articular incarceration of the epicondylar muscles; a tight digastric structure made up of the epicondyle, its epicondylar muscular attachments above and the periosteum and triceps layer detached from the humeral diaphysis below, lies in the humeroulnar joint and resists any attempt at reduction of the elbow dislocation.

5. Type-V: entrapment of the epicondyle in the joint with associated elbow dislocation or subluxation which may spontaneously reduce and mask the incarceration.
All of our patients underwent open reduction internal fixation of the medial epicondyle fracture. Average time between injury and indexed surgery was 2.5 days ( $S D=1.73$, range $0-12$ days). A medial approach to the elbow through an incision just posterior to the epicondyle was performed. The ulnar nerve was always isolated and protected. The medial epicondylar fragment was retrieved, anatomically reduced and fixed with two $\mathrm{K}$ wires in five cases (Fig 1), tension band wiring in two cases. Single or double $4 \mathrm{~mm}$ cannulated screws were implant of choice in 10 and three cases respectively (Fig 2, 3). After fracture repair, ulnar nerve was examined in their typical posteromedial location for subluxation and potential hardware irritation. The ulnar nerve was transposed during indexed surgery in three cases. Post-operative immobilization of the elbow was at flexed $90^{\circ}$ and neutral pronosupination of the forearm for a mean of 11 days (range 6-21) followed with elbow mobilization. Removal of $\mathrm{K}$ wires was performed between 4 to 8 weeks postoperatively and between 4 to 8 months in case of the screws and TBWs.

At postoperative clinic visits, we concluded detailed physical and radiographic examination. The Mayo Clinic performance index for the elbow was used for functional assessment in 4 areas: pain (maximum 45 points, from no pain to severe pain), stability (maximum 10 points, from stable to grossly unstable), range of movement (maximum 20 points, from $>100^{\circ}$ to $<50^{\circ}$ ), and activities of daily living (maximum 25 points). Score greater than 90 was considered excellent, 75-89 good, 60-74 fair, less than 60 poor. ${ }^{6}$ Other studies have used the Mayo elbow score for children and adolescents., Plain radiography was used to assess the union, detect pseudarthrosis or ectopic calcification. Any postoperative complications like ulnar nerve palsy, heterotrophic ossification, cubitus varus or valgus, and wound or implant-related were documented. Details of the patients are presented in Table 1.

\section{RESULTS:}

Mean follow up was 8.75 months (range 4-20). The fracture was fall related in all of our cases. The most common mechanism of injury was a fall from height on the outstretched hand with the elbow extended or partially flexed. Thirteen $(65 \%)$ were boys and 7 (35\%) were girls. Average age of children was 10.8 years ( $S D=2.3$, range $8-16)$. Twelve (60\%) fractures were associated with an elbow dislocation out of which five cases reduced spontaneously 


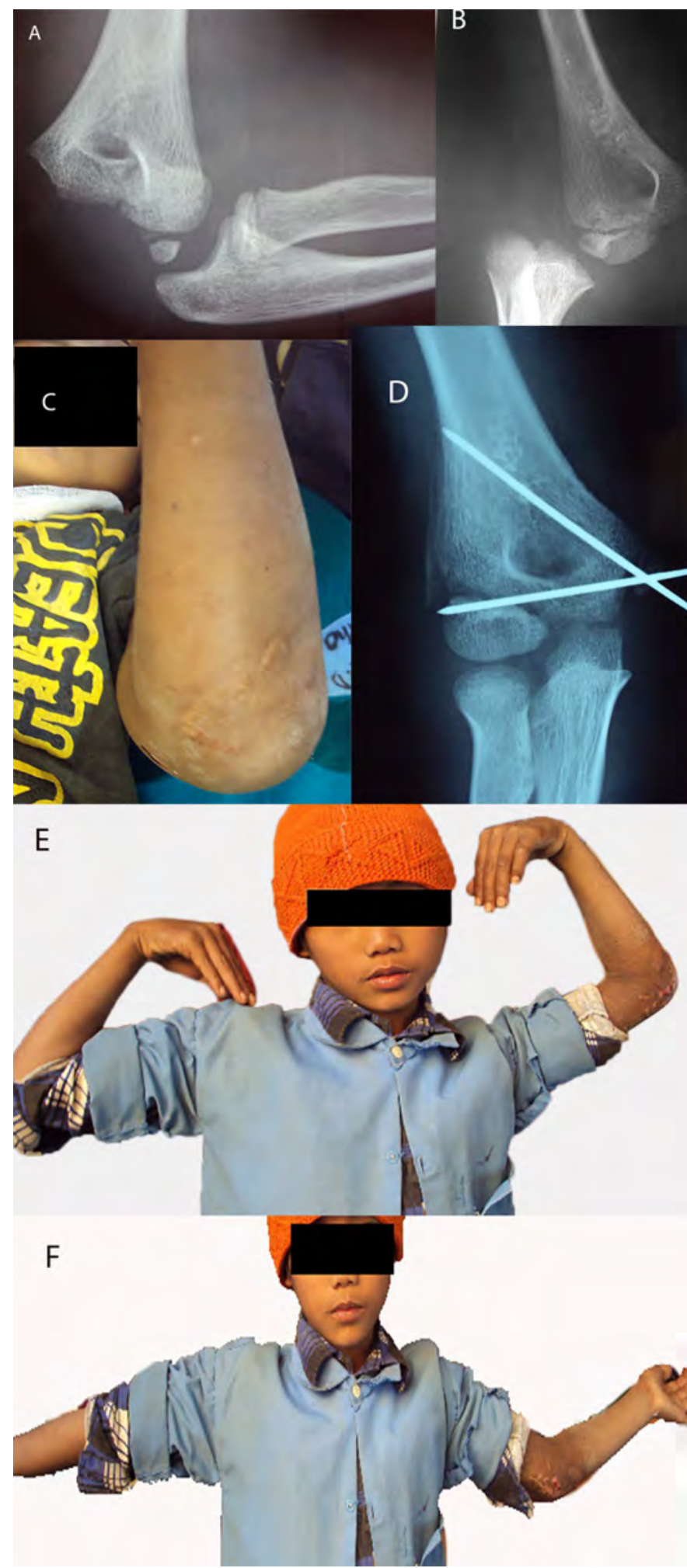

Fig 1: A,B. Representative pre-reduction radiographs (patient 1) showing a displaced medial epicondyle fracture in association with a posterolateral elbow dislocation; C. Injured elbow; D. 2 months postoperative radiographs showing fracture by $2 k$ wires. Note mild lateral heterotrophic ossification; Clinical picture showing $95^{\circ}$ flexion and extension lag of $40^{\circ}$.

and remaining 15 elbows were reduced either in Emergency Department or in operating room. The dominant elbow was injured in $14(70 \%)$ patients. The injuries were categorized in: six type II, nine type III, two type IV and three type V.

Three cases had incarceration of medial epicondyle after elbow dislocation that were surgically retrieved. Two children had ulnar nerve dysfunction among which one (patient 16) had concomitant medial condyle fracture who underwent external neurolysis and Kirschner wire fixation simultaneously.

At final follow-up, all patients had clinical and radiographic signs of healing. There was no radiographic evidence of loss of reduction at interval or final follow-up. Clinically, there were no cases of residual deformity or valgus instability. Average arc of motion of elbow was $80^{\circ}$ to $121^{\circ}$. Our patients had mean forearm rotation from $83^{\circ}$ supinations to $84^{\circ}$ pronation. Average Mayo elbow score was 93.5 $(S D=4.89)$. Three patients developed postoperative ulnar nerve neuropraxia, which recovered at time of final follow up. Despite screw removal, the patient who had preoperative ulnar symptoms and underwent ulnar nerve neurolysis in the index procedure (patient 15) had intermittent residual numbness and tingling in the little and ring fingers and mild weakness of the little and ring fingers at final follow up (13 months). The symptoms were mild enough that and family decided not to seek intervention. One patient developed mild heterotrophic ossification but did not require any additional surgical intervention and had forearm rotation of $80^{\circ}$ supination, $75^{\circ}$ pronation and elbow arc of $15^{\circ}-100^{\circ}$. Radiographically, union was achieved in all cases. Mean consolidation time was 4.5 weeks (range 3.4-8). Five cases had a fibrous union with a gap three $\mathrm{mm}$ between the medial epicondyle and the distal humeral metaphysis with no obvious change on valgus stress views. No cases of cubitus valgus more than $10^{\circ}$ were noticed in our patients.

\section{DISCUSSION:}

Medial epicondyle is a traction apophysis of distal humerus which is constantly under pull during various elbow motions by the strong flexor pronator muscles and the strong medial collateral ligament. Whenever a valgus force is applied with elbow extended or partially flexed, apophyseal avulsion of medial epicondyle and elbow dislocation occurs. ${ }^{9}$ Such an injury occurs as a result of tear of the capuloligamentous and anteromedial soft tissues. The valgus stress test complements a good way to detect and evaluate the extent of elbow instability.

In case of children, the bony constraints of the elbow are not fully developed, the stability of the elbow depends mainly on the soft tissue integrity. As a result, elbow dislocations in children will 

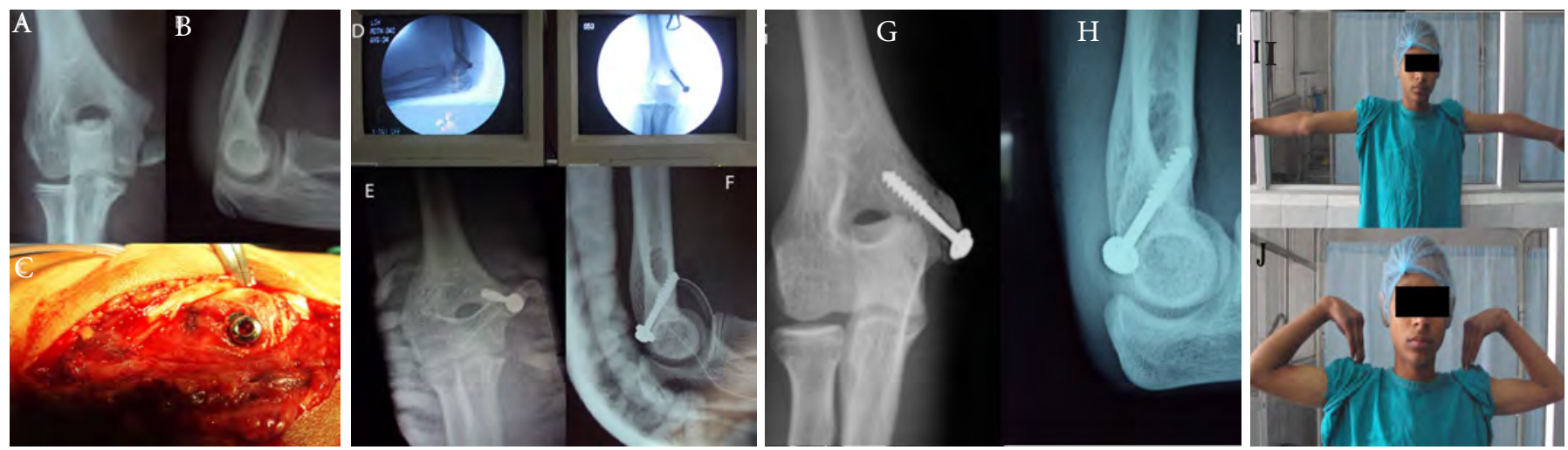

Fig 2: A,B. Antero-posterior and lateral X ray showing (patient 3) medial epicondylar fracture; $C$. Intraoperative picture showing a screw fixation following valgus stress test uncovering a postero-lateral dislocation of the elbow; D. Peroperative fluoroscopic images with anatomic reduction and fixation with screw. $\mathbf{E}, \mathbf{F}$. Immediate postopreative X-rays. G, $\mathbf{H}$. 9 months follow up radiographs showing bony healing of avulsed medial epicondylar fragment; I,J. No deformity appeared in the elbow region at the final follow up, while full movement of the elbow was restored completely.
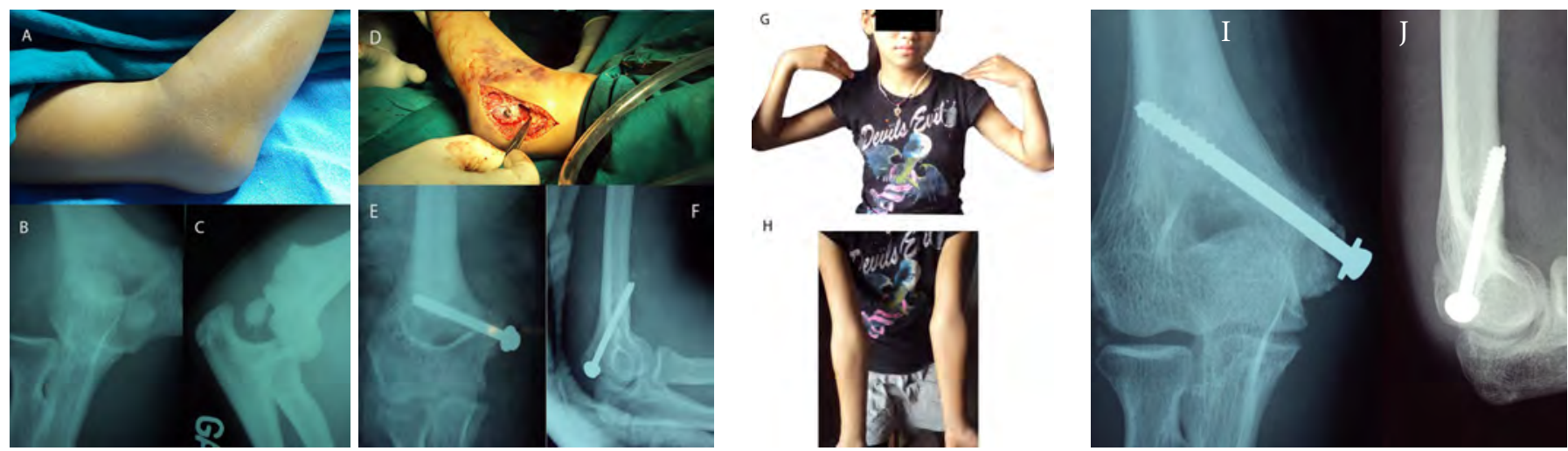

Fig 3: A. 9year-old girl(Patient 19) who had a medial epicondyle fracture type V; B,C. Dynamic antero-posterior radiograph (valgus stress test) showing dislocation with entrapment of the medial epicondyle; $\boldsymbol{D}$. Anatomic reduction of the epicondyle and screw fixation; note the importance of the soft tissue damage in the antero-medial aspect of the joint; $\boldsymbol{E}, \boldsymbol{F}$. Postoperative radiographs showing anatomic reduction with screw in situ; $\mathbf{G , H}$. Clinical results were graded as excellent according to the Mayo Elbow Performance Score, with no instability or impairment of range of motion 9 months after surgery; I,J. Healed medial epicondyle radiographs (9 months).

causes extensive damage of the soft tissue structures of the elbow. Damage to the medial stabilizing soft tissues structures of the elbow rather than the medial epicondyle displacement has a far greater consequence on joint stability and outcome. ${ }^{10,11}$ Woods and Tullos described the importance of assessing instability by using the "gravity valgus with or without stress" test. ${ }^{12}$

In our series, valgus stress test was performed in operating room under anaesthesia and was found to ba a reliable diagnostic test and a good indication for surgical fixation in the presence of signifcant acute medial elbow instability in all cases of medial epicondyle.

Treatment of medial epicondyle fractures is controversial. Hines et al. recommended that all fractures with displacements over two mm required fixation. ${ }^{1}$ Absolute indications for open reduction and internal fixation of incarcerated medial epicondylar fractures into the elbow joint, suspected entrapment and ulnar nerve dysfunction, marked instability, or open fracture. There is ongoing debate about the management of cases that do not meet the above indications based on the degree of displacement, handedness, and athletic and performance demands. Chronic elbow instability following non operative treatment was described by Woods and Tullos, and Schwab et al. ${ }^{11,12}$ Case and Hennrikus reported excellent results following open reduction and rigid internal fixation of acute displaced medial epicondyle fractures in adolescent athletes. ${ }^{15}$ In our series, elbow stability was achieved in all patients as a result of surgical fixation. For some Orthopaedic surgeons, our treatment may be too much surgical oriented. Our study allows to imply that the clinical outcomes as measured by range of motion and Mayo elbow score after surgery is very satisfactory. The consistent good results were achieved by operated fixation as reported by Hines et al., Lee et al. and Fowles et al. along with our own series would advocate surgical intervention when there is a displacement. ${ }^{1,4,16}$ Operative management allows for anatomic reduction internal fixation of the fragment and incase of incarceration, removal 


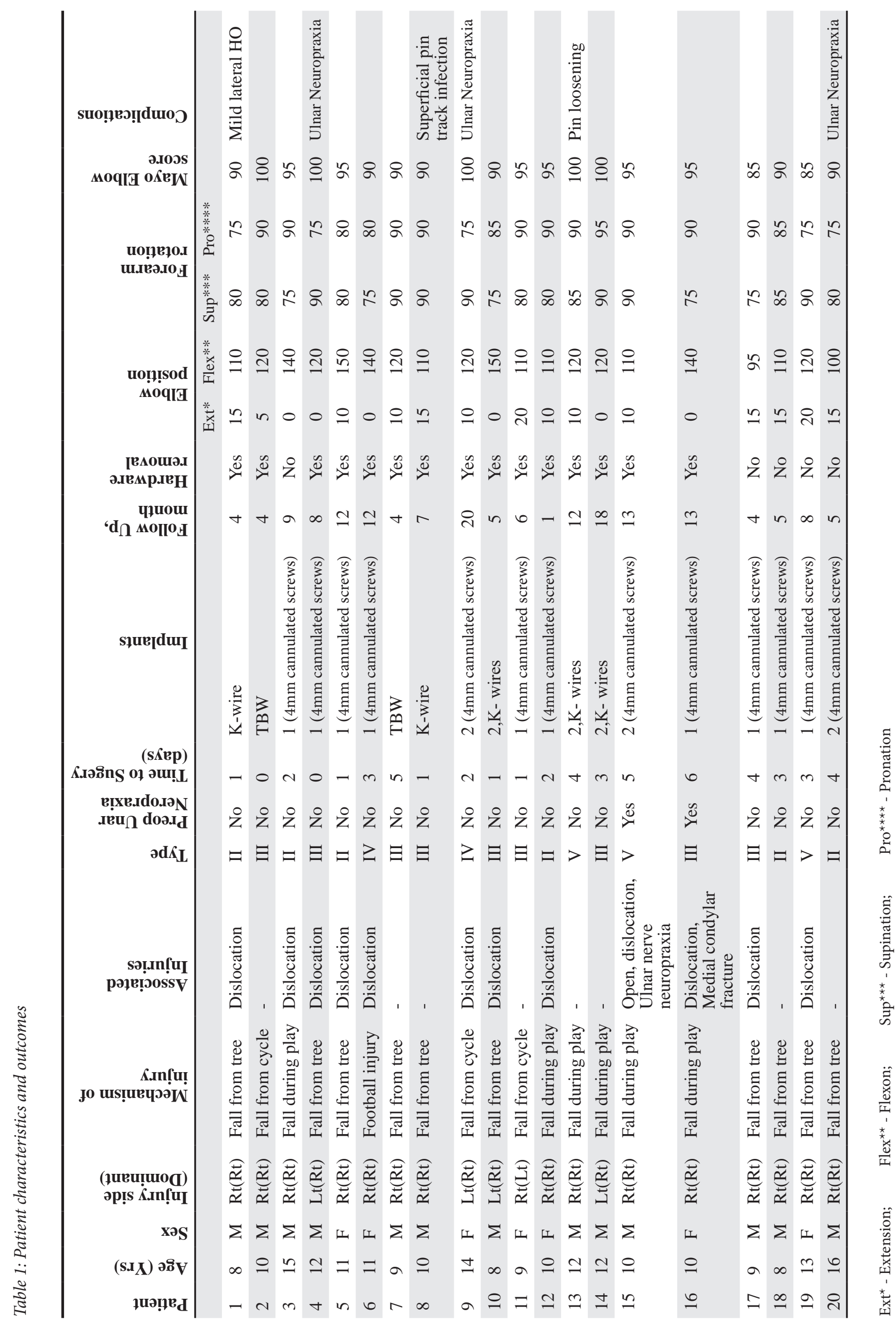


of the offending fragment under direct visualization followed by stabilization. Surgical reduction and fixation of medial epicondyle along with its strong flexor pronator muscle origin and medial collateral ligament may contribute to critical restraint against valgus instability and allow for early elbow movements.

However, the findings of other variables may be affected by smaller sample size, which was one of the limitations of our study and a point that could be evaluated in future studies.

\section{REFERENCES:}

1. Hines RF, Herndon WA, Evans JP. Operative treatment of medial epicondyle fractures in children. Clin Orthop Relat Res. 1987;223:170-4.

2. Beaty JH, Kasser JR. The elbow-physeal fractures, apophyseal injuries of the distal humerus, osteonecrosis of the trochlea, and T-condylar fractures. In: Beaty JH, Kasser JR, editors. Rockwood \& Wilkins' fractures in children. 7th ed. Philadelphia:Wolters Kluwer Health; 2012.

3. Patel NM, Ganley TJ. Medial epicondyle fractures of the humerus: how to evaluate and when to operate. J Pediatr Orthop. 2012; 32(1):S10-3. doi: 10.1097/ BPO.0b013e31824b2530.

4. Lee $\mathrm{HH}$, Shen $\mathrm{HC}$, Chang JH, Lee $\mathrm{CH}, \mathrm{Wu}$ SS. Operative treatment of displaced medial epicondyle fractures in children and adolescents. J Shoulder Elbow Surg. 2005;14(2):178-85.

5. Smith FM. Medial epicondyle injuries. JAMA. 1950;142:396-402.

6. Morrey BF, An KN, Chao EYS. Functional evaluation of the elbow. In: Morrey BF, ed. The Elbow and Its Disorders. 2nd ed. Philadelphia:W.B. Saunders; 1993.

7. Moraleda L, Valencia M, Barco R, González-Moran G. Natural history of unreduced Gartland type-II supracondylar fractures of the humerus in children: a two to thirteen-year follow-up study. J Bone Joint Surg Am. 2013;95(1):28-34.

8. Bowakim J, Marti R, Curto A. Elbow septic arthritis in

\section{CONCLUSIONS:}

Fractures of the medial epicondyle of the humerus are often associated with elbow dislocation. The valgus stress test performed at the time of surgery for all epicondyle fractures regardless of the degree of its displacement can unmask true nature of extensive medial soft tissue injuries. In view of good results and presumably lower rate of nonunion and joint instability, we recommend operative intervention as a good management of these injuries and results in an anatomic reduction, a solid bone union and prevents valgus instability.

children: clinical presentation and management. J Pediatr Orthop B. 2010;19(3):281-4.

9. Kilfoyle RM. Fracture of the medial condyle and epicondyle of the elbow in children. Clin Orthop Relat Res. 1965;41:43-50.

10. Lechevallier J, Lefort J. Les Fractures du coude : les fractures de l'épitrochlée. Rev Chir Orthop. 1987;73:441-7.

11. Schwab GH, Bennet JB, Woods GW, Tullos HS Biomechanics of elbow instability: the role of the medial collateral ligament. Clin Orthop Relat Res. 1980;146:42-52.

12. Woods GW, Tullos HS. Elbow instability and medial epicondyle fractures. Am J Sports Med. 1977;5:23-30. doi: $10.1177 / 036354657700500105$

13. Josefsson PO, Danielsson LG. Epicondylar elbow fracture in children. 35-year follow-up of 56 unreduced cases. Acta Orthop Scand. 1986;57:313-5. doi: $10.3109 / 17453678608994399$.

14. Kobayashi Y, Oka Y, Ikeda M, Munesada S. Avulsion fracture of the medial and lateral epicondyles of the humerus. J Shoulder Elbow Surg. 2000;9(1):59-64.

15. Case SL, Hennrikus WL. Surgical treatment of displaced medial epicondyle fractures in adolescent athletes. Am J Sports Med. 1997;25(5):682-6.

16. Fowles JV, Slimane N, Kassab MT (1990) Elbow dislocation with avulsion of the medial humeral epicondyle. J Bone Joint Surg Br. 72:102-104. 